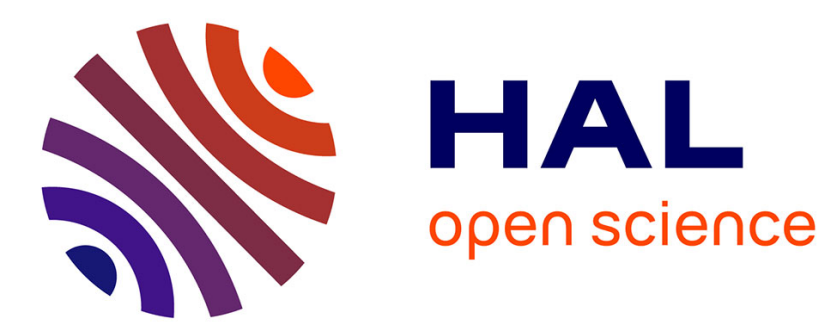

\title{
Valeur pronostique du volume métabolique initial dans le cancer épidermoïde de l'œesophage
}

Charles Lemarignier

\section{To cite this version:}

Charles Lemarignier. Valeur pronostique du volume métabolique initial dans le cancer épidermoïde de l'œesophage. Médecine humaine et pathologie. 2014. dumas-01060828

\section{HAL Id: dumas-01060828 https://dumas.ccsd.cnrs.fr/dumas-01060828}

Submitted on 4 Sep 2014

HAL is a multi-disciplinary open access archive for the deposit and dissemination of scientific research documents, whether they are published or not. The documents may come from teaching and research institutions in France or abroad, or from public or private research centers.
L'archive ouverte pluridisciplinaire HAL, est destinée au dépôt et à la diffusion de documents scientifiques de niveau recherche, publiés ou non, émanant des établissements d'enseignement et de recherche français ou étrangers, des laboratoires publics ou privés. 
FACULTÉ MIXTE DE MÉDECINE ET DE PHARMACIE DE ROUEN

ANNÉE 2014

$\mathrm{N}^{\circ}$

\section{THÈSE POUR LE DOCTORAT EN MÉDECINE}

(Diplôme d'État)

Par Charles Lemarignier

Né le 6 février 1986 à Mont-Saint-Aignan

Présentée et soutenue publiquement le 25 mars 2014

\section{VALEUR PRONOSTIQUE DU VOLUME MÉTABOLIQUE INITIAL DANS LE CANCER ÉPIDERMOÏDE DE L'EESOPHAGE.}

DIRECTEUR DE THÈSE : Professeur Pierre Vera

MEMBRES DU JURY : Professeur Pierre Michel

Professeur Bernard Dubray

Docteur Frédéric Di Fiore 


\section{ANNÉE UNIVERSITAIRE 2013 - 2014 \\ U.F.R. DE MÉDECINE-PHARMACIE DE ROUEN}

DOYEN :

Professeur Pierre FREGER

ASSESSEURS :

Professeur Michel GUERBET

Professeur Benoit VEBER

Professeur Pascal JOLY

DOYENS HONORAIRES :

Professeurs J. BORDE - Ph. LAURET - H. PIGUET - C. THUILLEZ

PROFESSEURS HONORAIRES : MM. M.-P. AUGUSTIN - J. ANDRIEU-GUITRANCOURT M. BENOZIO - J. BORDE - Ph. BRASSEUR - R. COLIN E. COMOY - J. DALION - DESHAYES - C. FESSARD - J.P. FILLASTRE - P. FRIGOT - J. GARNIER - J. HEMET B. HILLEMAND - G. HUMBERT - J.-M. JOUANY - R. LAUMONIER - Ph. LAURET - M. LE FUR - J.-P. LEMERCIER - J.-P. LEMOINE - Mlle MAGARD - MM. B. MAITROT - M. MAISONNET - F. MATRAY - P. MITROFANOFF - Mme A.-M. ORECCHIONI - MM. P. PASQUIS - H. PIGUET - M. SAMSON - Mme SAMSONDOLLFUS - MM. J.-C. SCHRUB - R. SOYER - B. TARDIF - J. TESTART - J.M. THOMINE - C. THUILLEZ - P. TRON - C. WINCKLER - L.-M. WOLF

\section{I - MÉDECINE}

\section{PROFESSEURS}

M. Frédéric ANSELME

Mme Isabelle AUQUIT AUCKBUR

M. Bruno BACHY (Surnombre)

M. Fabrice BAUER

Mme Soumeya BEKRI

M. Jacques BENICHOU

médicale

M. Jean-Paul BESSOU

vasculaire

\author{
HCN Cardiologie \\ $\mathrm{HCN}$ Chirurgie Plastique \\ $\mathrm{HCN}$ Chirurgie pédiatrique \\ $\mathrm{HCN}$ Cardiologie \\ $\mathrm{HCN}$ Biochimie et Biologie Moléculaire \\ $\mathrm{HCN}$ Biostatistiques et informatique
}

$\mathrm{HCN}$ Chirurgie thoracique et cardio- 
Mme Françoise BEURET-BLANQUART (Surnombre) CRMPR Médecine physique et de réadaptation

M. GuY BONMARCHAND

M. Olivier BOYER

M. Jean-François CAILLARD (Surnombre)

M. François CARON

M. Philippe CHASSAGNE

M. Vincent COMPERE

chirurgicale

M. Antoine CUVELIER

M. Pierre CZERNICHOW

santé

M. Jean-Nicolas DACHER

M. Stéfan DARMONI

de communication

M. Pierre DECHELOTTE

Mme Danièle DEHESDIN (Surnombre)

M. Jean DOUCET

- Gériatrie.

M. Bernard DUBRAY

M. Philippe DUCROTTE

M. Frank DUJARDIN

Traumatologique

M. Fabrice DUPARC

Orthopédique et Traumatologique

M. Bertrand DUREUIL

chirurgicale

Mme Hélène ELTCHANINOFF

M. Thierry FREBOURG

M. Pierre FREGER

M. Jean François GEHANNO

M. Emmanuel GERARDIN

Mme Priscille GERARDIN

M. Michel GODIN

M. Guillaume GOURCEROL

M. Philippe GRISE

M. Didier HANNEQUIN
HCN Réanimation médicale

UFR Immunologie

HCN Médecine et santé au Travail

$\mathrm{HCN}$ Maladies infectieuses et tropicales

HB Médecine interne (Gériatrie)

$\mathrm{HCN}$ Anesthésiologie et réanimation

HB Pneumologie

$\mathrm{HCH}$ Epidémiologie, économie de la

HCN Radiologie et Imagerie Médicale

$\mathrm{HCN}$ Informatique Médicale/Techniques

HCN Nutrition

HCN Oto-Rhino-Laryngologie

HB Thérapeutique/Médecine - Interne

CB Radiothérapie

HCN Hépato - Gastro - Entérologie

HCN Chirurgie Orthopédique -

$\mathrm{HCN}$ Anatomie - Chirurgie

$\mathrm{HCN}$ Anesthésiologie et réanimation

$\mathrm{HCN}$ Cardiologie

UFR Génétique

$\mathrm{HCN}$ Anatomie/Neurochirurgie

HCN Médecine et Santé au Travail

$\mathrm{HCN}$ Imagerie Médicale

HCN Pédopsychiatrie

HB Néphrologie

HCN Physiologie

$\mathrm{HCN}$ Urologie

$\mathrm{HCN}$ Neurologie 
M. Fabrice JARDIN

M. Luc-Marie JOLY

M. Pascal JOLY

M. Jean-Marc KUHN

métaboliques

Mme Annie LAQUERRIERE

M. Vincent LAUDENBACH

chirurgicale

M. Joël LECHEVALLIER

M. Hervé LEFEBVRE

métaboliques

M. Thierry LEQUERRE

M. Eric LEREBOURS

Mme Anne-Marie LEROI

M. Hervé LEVESQUE

Mme Agnès LIARD-ZMUDA

M. Pierre Yves LITZLER

M. Bertrand MACE

cytogénétique

M. David MALTETE

M. Christophe MARGUET

Mme Isabelle MARIE

M. Jean-Paul MARIE

M. LoÏc MARPEAU

M. Stéphane MARRET

Mme Véronique MERLE

M. Pierre MICHEL

M. Francis MICHOT

M. Bruno MIHOUT (Surnombre)

M. Jean-François MUIR

M. Marc MURAINE

M. Philippe MUSETTE

M. Christophe PEILLON

M. Jean-Marc PERON

faciale

M. Christian PFISTER

M. Jean-Christophe PLANTIER
CB Hématologie

HCN Médecine d'urgence

HCN Dermato - vénéréologie

HB Endocrinologie et maladies

HCN Anatomie cytologie pathologiques

$\mathrm{HCN}$ Anesthésie et réanimation

$\mathrm{HCN}$ Chirurgie infantile

HB Endocrinologie et maladies

HB Rhumatologie

HCN Nutrition

HCN Physiologie

HB Médecine interne

HCN Chirurgie Infantile

$\mathrm{HCN}$ Chirurgie Cardiaque

$\mathrm{HCN}$ Histologie, embryologie,

$\mathrm{HCN}$ Neurologie

HCN Pédiatrie

HB Médecine Interne

HCN ORL

HCN Gynécologie - obstétrique

$\mathrm{HCN}$ Pédiatrie

$\mathrm{HCN}$ Epidémiologie

HCN Hépato - Gastro - Entérologie

$\mathrm{HCN}$ Chirurgie digestive

HCN Neurologie

HB Pneumologie

$\mathrm{HCN}$ Ophtalmologie

HCN Dermatologie - Vénéréologie

$\mathrm{HCN}$ Chirurgie générale

HCN Stomatologie et chirurgie maxillo-

HCN Urologie

HCN Bactériologie - Virologie 
M. Didier PLISSONNIER

M. Bernard PROUST

M. François PROUST

Mme Nathalie RIVES

développement et de la reproduction

M. Jean-Christophe RICHARD (Mise en disponibilité) d'urgence

M. Horace ROMAN

M. Jean-Christophe SABOURIN

M. Guillaume SAVOYE

Mme Céline SAVOYE-COLLET

Mme Pascale SCHNEIDER

M. Michel SCOTTE

Mme Fabienne TAMION

Mme Florence THIBAUT

M. Luc THIBERVILLE

M. Christian THUILLEZ

M. Hervé TILLY

M. François TRON (Surnombre)

M. Jean-Jacques TUECH

M. Jean-Pierre VANNIER

M. Benoît VEBER

chirurgicale

M. Pierre VERA

l'image

M. Eric VERIN

réadaptation

M. Eric VERSPYCK

M. Olivier VITTECOQ

M. Jacques WEBER

\section{MAÎTRES DE CONFÉRENCES}

Mme Noëlle BARBIER-FREBOURG

M. Jeremy BELLIEN

Mme Carole BRASSE LAGNEL

M. Gérard BUCHONNET
$\mathrm{HCN}$ Chirurgie vasculaire

$\mathrm{HCN}$ Médecine légale

$\mathrm{HCN}$ Neurochirurgie

$\mathrm{HCN}$ Biologie et médecine du

HCN Réanimation Médicale, Médecine

HCN Gynécologie Obstétrique

HCN Anatomie - Pathologie

HCN Hépato - Gastro

$\mathrm{HCN}$ Imagerie Médicale

$\mathrm{HCN}$ Pédiatrie

$\mathrm{HCN}$ Chirurgie digestive

$\mathrm{HCN}$ Thérapeutique

HCN Psychiatrie d'adultes

HCN Pneumologie

HB Pharmacologie

CB Hématologie et transfusion

UFR Immunologie

HCN Chirurgie digestive

$\mathrm{HCN}$ Pédiatrie génétique

HCN Anesthésiologie Réanimation

CB Biophysique et traitement de

CRMPR Médecine physique et de

HCN Gynécologie obstétrique

HB Rhumatologie

HCN Physiologie
HCN Bactériologie - Virologie

HCN Pharmacologie

$\mathrm{HCN}$ Biochimie

HCN Hématologie 
Mme Mireille CASTANET

Mme Nathalie CHASTAN

Mme Sophie CLAEYSSENS

M. Moïse COEFFIER

M. Stéphane DERREY

M. Eric DURAND

M. Manuel ETIENNE

M. Serge JACQUOT

M. Joël LADNER

santé

M. Jean-Baptiste LATOUCHE

M. Thomas MOUREZ

M. Jean-François MENARD

Mme Muriel QUILLARD

M. Vincent RICHARD

M. Francis ROUSSEL

cytogénétique

Mme Pascale SAUGIER-VEBER

Mme Anne-Claire TOBENAS-DUJARDIN

M. Pierre Hugues VIVIER

\section{PROFESSEURS AGRÉGÉS OU CERTIFIÉS}

Mme Dominique LANIEZ

Mme Cristina BADULESCU
HCN Pédiatrie

HCN Physiologie

$\mathrm{HCN}$ Biochimie et biologie moléculaire

HCN Nutrition

$\mathrm{HCN}$ Neurochirurgie

$\mathrm{HCN}$ Cardiologie

HCN Maladies infectieuses et tropicales

UFR Immunologie

$\mathrm{HCN}$ Epidémiologie, économie de la

UFR Biologie Cellulaire

$\mathrm{HCN}$ Bactériologie

HCN Biophysique

HCN Biochimie et Biologie moléculaire

UFR Pharmacologie

HCN Histologie, embryologie,

HCN Génétique

$\mathrm{HCN}$ Anatomie

HCN Imagerie Médicale

$\begin{array}{ll}\text { UFR } & \text { Anglais } \\ \text { UFR } & \text { Communication }\end{array}$




\section{II - PHARMACIE}

\section{PROFESSEURS}

M. Thierry BESSON

M. Jean-Jacques BONNET

M. Roland CAPRON (PU-PH)

M. Jean COSTENTIN (Professeur émérite)

Mme Isabelle DUBUS

M. LoÏc FAVENNEC (PU-PH)

M. Jean Pierre GOULLE

M. Michel GUERBET

M. Olivier LAFONT

Mme Isabelle LEROUX

M. Paul MULDER

Mme Martine PESTEL-CARON (PU-PH)

Mme Elisabeth SEGUIN

M. Rémi VARIN (PU-PH)

M. Jean-Marie VAUGEOIS

M. Philippe VERITE

\section{MAÎTRES DE CONFÉRENCES}

Mme Cécile BARBOT

Mme Dominique BOUCHER

M. Frédéric BOUNOURE

M. Abdeslam CHAGRAOUI

M. Jean CHASTANG

Mme Marie Catherine CONCE-CHEMTOB

Mme Elizabeth CHOSSON

Mme Cécile CORBIERE

M. Eric DITTMAR

Mme Nathalie DOURMAP

Mme Isabelle DUBUC
Chimie Thérapeutique

Pharmacologie

Biophysique

Pharmacologie

Biochimie

Parasitologie

Toxicologie

Toxicologie

Chimie organique

Physiologie

Sciences du médicament

Microbiologie

Pharmacognosie

Pharmacie Hospitalière

Pharmacologie

Chimie analytique
Chimie Générale et Minérale

Pharmacologie

Pharmacie Galénique

Physiologie

Biomathématiques

Législation pharmaceutique et économie de la santé

Botanique

Biochimie

Biophysique

Pharmacologie

Pharmacologie 
M. Abdelhakim ELOMRI

M. François ESTOUR

M. Gilles GARGALA (MCU-PH)

Mme Najla GHARBI

Mme Marie-Laure GROULT

M. Hervé HUE

Mme Laetitia LE GOFF

Mme Hong LU

Mme Sabine MENAGER

Mme Christelle MONTEIL

M. Mohamed SKIBA

Mme Malika SKIBA

Mme Christine THARASSE

M. Frédéric ZIEGLER

\section{PROFESSEUR CONTRACTUEL}

Mme Elizabeth DE PAOLIS
Pharmacognosie

Chimie Organique

Parasitologie

Chimie analytique

Botanique

Biophysique et Mathématiques

Parasitologie Immunologie

Biologie

Chimie organique

Toxicologie

Pharmacie Galénique

Pharmacie Galénique

Chimie thérapeutique

Biochimie

Anglais

\section{ATTACHÉS TEMPORAIRES D'ENSEIGNEMENT ET DE RECHERCHE}

M. Imane EL MEOUCHE

Mme Juliette GAUTIER

M. Romy RAZAKANDRAINIBE
Bactériologie

Galénique

Parasitologie 


\section{PROFESSEUR}

M. Jean-Loup HERMIL

UFR Médecine générale

PROFESSEURS ASSOCIÉS À MI-TEMPS
M. Pierre FAINSILBER
UFR Médecine générale
M. Alain MERCIER
UFR Médecine générale
M. Philippe NGUYEN THANH
UFR Médecine générale

MAÎTRE DE CONFÉRENCES ASSOCIÉS À MI-TEMPS :

M. Emmanuel LEFEBVRE

Mme Elisabeth MAUVIARD

Mme Marie Thérèse THUEUX

Mme Yveline SERVIN

$\begin{array}{ll}\text { UFR } & \text { Médecine générale } \\ \text { UFR } & \text { Médecine générale } \\ \text { UFR } & \text { Médecine générale } \\ \text { UFR } & \text { Médecine Générale }\end{array}$

UFR Médecine générale

UFR Médecine générale

UFR Médecine Générale

CHEF DES SERVICES ADMINISTRATIFS : Mme Véronique DELAFONTAINE

HCN - Hôpital Charles Nicolle

HB - Hôpital de BOIS-GUILLAUME

CB - Centre HENRI BECQUEREL

CHS - Centre Hospitalier Spécialisé du Rouvray

CRMPR - Centre Régional de Médecine Physique et de Réadaptation 
Mme Cécile BARBOT

M. Thierry BESSON

M. Roland CAPRON

M. Jean CHASTANG

Mme Marie-Catherine CONCE-CHEMTOB

Mme Elisabeth CHOSSON

M. Jean-Jacques BONNET

Mme Isabelle DUBUS

M. Loïc FAVENNEC

M. Michel GUERBET

M. Olivier LAFONT

Mme Isabelle LEROUX-NICOLLET

Mme Martine PESTEL-CARON

Mme Elisabeth SEGUIN

M. Mohamed SKIBA

M. Philippe VERITE
Chimie Générale et Minérale

Chimie thérapeutique

Biophysique

Mathématiques

Législation, Economie de la Santé

Botanique

Pharmacodynamie

Biochimie

Parasitologie

Toxicologie

Chimie organique

Physiologie

Microbiologie

Pharmacognosie

Pharmacie Galénique

Chimie analytique 


\section{MAÎTRES DE CONFÉRENCES}

M. Sahil ADRIOUCH

Mme Gaëlle BOUGEARD-DENOYELLE

Mme Carine CLEREN

Mme Pascaline GAILDRAT

M. Antoine OUVRARD-PASCAUD

Mme Isabelle TOURNIER

PROFESSEURS DES UNIVERSITÉS

M. Serguei FETISSOV

Mme Su RUAN
Biochimie et biologie moléculaire

(Unité Inserm 905)

Biochimie et biologie moléculaire (UMR 1079)

Neurosciences (Néovasc)

Génétique moléculaire humaine (UMR 1079)

Physiologie (Unité Inserm 1076)

Biochimie (UMR 1079)
Physiologie (Groupe ADEN)

Génie Informatique 
Par délibération en date du 3 mars 1967, la faculté a arrêté que les opinions émises dans les dissertations qui lui seront présentées doivent être considérés comme propres à leurs auteurs et qu'elle n'entend leur donner aucune approbation ni improbation. 


\section{Remerciements}

Au Professeur Pierre Vera, pour m'avoir confié ce sujet et avoir accepté d'être mon directeur de thèse. Pour avoir été toujours disponible et réactif sur ce projet. Pour avoir dirigé mon encadrement pendant ces 4 années d'internat et m'avoir transmis ses connaissances en médecine nucléaire.

Au professeur Pierre Michel, pour m'avoir permis de traiter ce sujet, pour avoir su nous dire d'aller de l'avant et de passer à l'étape suivante au moment opportun. Pour sa gentillesse et ses remarques constructives.

Au Docteur Frédéric Di Fiore, pour son aide, son encadrement rapproché sur ce travail dans le cadre de nos réunions du lundi après-midi.

Au Professeur Bernard Dubray, pour son aide précieuse dans l'analyse statistique et sa bonne connaissance du sujet et de la discipline. Pour m'avoir accueilli dans son service.

Au Docteur Ahmed Ben Youcef, pour sa connaissance de la pathologie et des modalités de traitements.

À Monsieur Sébastien Hapdey, pour sa sympathie, son soutien, ses corrections justes et efficaces.

À Madame Isabelle Gardin, pour avoir soutenu et encouragé ce travail, pour m'avoir permis de le présenter à trois reprises aux réunions de recherche.

À Charline Marre, pour les soirées et les week-ends de recueil de données.

À Pierrick Gouel et Romain Modzelewski, pour leur aide précieuse dans la réalisation de ce travail.

Au Docteur Lamyaa Nkhali, pour son aide dans la description du volume irradié. 
Au Docteur Joseph Le Cloirec, pour sa connaissance encyclopédique de la médecine nucléaire et de la médecine en général, pour sa disponibilité et sa patience. Pour m'avoir transmis au quotidien la passion et l'amour de mon métier.

Au Docteur Agathe Edet-Sanson, pour m'avoir transmis ses connaissances en médecine nucléaire, pour sa bienveillance quasi maternelle.

Au Docteur Stéphanie Becker, pour sa présence, sa rigueur et pour m'avoir transmis ses connaissances en médecine nucléaire

Au Docteur Stéphane Gaucher, dont l'intérêt pour la cardiologie nucléaire n'a d'égal que celui des objets d'art, de la musique et de la peinture, pour son autorité paternelle.

Au Docteur Mathieu Chastan, pour avoir assuré le rôle de « chef de clinique » à notre arrivé dans le service, pour sa disponibilité et sa proximité, pour nous avoir transmis ses connaissances en médecine nucléaire.

Au Docteur Sébastien Thureau, pour sa connaissance de la cancérologie, pour son écoute.

À mes co-internes de médecine nucléaire, Odré, dont les travaux de thèse ont inspiré les miens ; Anne-Charlotte, qui m'a accueilli dans le service et avec qui j'ai effectué dans la bonne humeur ma formation à Saclay; Jérémie, avec qui j'ai partagé mon premier stage de radiologie et mon premier stage de médecine nucléaire, nous avons formé un sacré duo ! Anne-Ségolène, pour sa constance et sa douceur ; Julien et Mathieu, pour l'intérêt qu'ils montrent déjà pour la médecine nucléaire.

À mes co-interne de radiologie, Laurent, Manuel, Samuel, Nacer, Valentin, Clément, Benjamin, Valérie, Hamel, et Nicolas, pour leur patience, leur inclination à partager leur savoir.

À mes co-internes de Becquerel Marion, Maxime, Ophélie, Simon.

À mes co-internes de radiothérapie, Lucie, Laureline, Albane, Thierry et François-Xavier, pour m'avoir encouragé lors de la dernière ligne droite. 
Judith, Jean, Alice et Pierre, toujours présent pour répondre à mes questions de radiopharmacie.

À Charlotte, Christelle, Claire, Dédé, Mireille, Muriel, Tiphanie, Véro, Arthur, Manu, Raph, Romain sans qui il n'y aurait pas d'image, Thierry et Francis pour leur gestion de l'équipe ; à Pascal qui nous radio-protège ; à Zorah, pour ses pâtisseries, Françoise, pour les discussions le soir à la fermeture du service, et Josiane, pour sa présence ; à Béa, Béné, Céline, Clémence, Corinne, Emilie, Maguy, Séverine, au secrétariat, toujours disponible et de bonne humeur.

Aux manip des services de radiologie de l'hôpital d'Evreux, de l'hôpital Charles Nicolle et du service de radiothérapie du Centre Henri Becquerel.

Au Docteur Christian Richard, pour avoir accompagné mes premiers pas d'interne.

Au Professeur Jean-Nicolas Dacher, pour sa grande gentillesse, son soutien, pour m'avoir accueilli dans son service et transmis son amour pour l'imagerie médicale.

Aux Docteurs Paule et François Kuntz, pour avoir accompagné la naissance de ma "vocation".

À Guillaume Armengol, pour avoir suivi avec intérêt les 10 années de mon parcours.

À Rodolphe Ménard, pour avoir fait grandir en moi l'intérêt pour l'imagerie médicale.

À Damien, pour son soutien dans la préparation de l'internat et les quarts de nuit en atlantique nord.

À Carl, pour son soutien quotidien et ses corrections.

À Hannah, pour la relecture de l'anglais.

À mes parents, Christine et François Lemarignier, pour m'avoir transmis leurs valeurs du travail et pour leur soutien infaillible. 
Table des matières

$\begin{array}{ll}\text { Remerciements } & 13\end{array}$

$\begin{array}{ll}\text { Table des matières } & 17\end{array}$

$\begin{array}{ll}\text { Table des illustrations } & 19\end{array}$

$\begin{array}{ll}\text { Avant-propos } & 21\end{array}$

$\begin{array}{ll}\text { Article original } & 27\end{array}$

Résumé - Abstract 29

Introduction - Introduction 31

Matériels et méthodes - Materials and methods 33

Résultats - Results 39

Discussion - Discussion $\quad 45$

Bibliographie - References 49

Conclusion $\quad 53$

$\begin{array}{ll}\text { Annexes } & 55\end{array}$ 


\section{Table des illustrations}

Figure 1 Organigramme

Flow-chart

Figure 2 Exemple de définition de volume

Examples of delineation

Figure 3 Courbes de survie

Kaplan-Meier survival curves

Table 1 Caractéristiques démographiques et cliniques des 67 patients

Demographic and clinical characteristics of the 67 patients

Table 2 Caractéristiques tumorales, devenir et suivi

Tumour characteristics, outcome and follow-up

Table 3 Paramètres TEP initiaux

Initial PET derived parameters

Table 4 Analyse multi-variée pour la survie sans maladie et la survie globale 
En l'absence de mention particulière, les notions développées dans l'avant-propos sont issues des référentiels nationaux suivants :

\section{Thésaurus National de Cancérologie Digestive}

Chapitre 1 : Cancer de l'œsophage. Version du 01/03/2013

Le Thésaurus est un travail collaboratif sous égide de la Fédération Francophone de Cancérologie Digestive (FFCD), de la Fédération Nationale des Centres de Lutte Contre le Cancer (FNCLCC), du Groupe Coopérateur multidisciplinaire en Oncologie (GERCOR), de la Société Française de Chirurgie Digestive (SFCD), de la Société Française de Radiothérapie Oncologique (SFRO) et de la Société Nationale Française de Gastroentérologie (SNFGE).

\section{CLes cancers en France en 2013.}

Institut National du Cancer www.e-cancer.fr

Collection État des lieux et des connaissances, ouvrage collectif édité par l'INCa, Boulogne-Billancourt, janvier 2014. 


\section{Avant-propos}

Avec 480000 nouveaux cas par an dans le monde et 4300 en France, le cancer de l'œsophage n'est pas une maladie excessivement fréquente. Il est ainsi moins fréquent que le cancer du poumon, de la prostate ou encore du colon ou de l'estomac. Il n'en reste pas moins une maladie particulièrement meurtrière, avec en France plus de 3000 morts par an, soit presque autant que le nombre de tués chaque année sur les routes. Ainsi le cancer de l'œsophage est un cancer de mauvais pronostic au même titre que les cancers du poumon ou de la plèvre, du foie, du pancréas, et du système nerveux central, et dont la survie à 5 ans n'excède pas $20 \%$. Il touche plus souvent l'homme que la femme avec un sex-ratio de 2,75. La principale plainte fonctionnelle incitant le patient à consulter est la dysphagie, et le cancer de l'œsophage est en général diagnostiqué tardivement, à un stade avancé chez des patients souvent déjà dénutris, c'est à dire avec une perte de poids supérieure à $10 \%$ du poids habituel sur les 6 derniers mois. Le diagnostic est fait sur les biopsies obtenues en fibroscopie œsogastroduodénale. Le carcinome épidermoïde reste le type histologique prédominant dans le monde et représente en France plus des trois quarts des types histologiques. Le principal facteur étiologique est l'intoxication alcoolo-tabagique. Cette intoxication est par ailleurs responsable chez ces patients de pathologies cardiaques, vasculaires ou respiratoires associées mais aussi de cancers associés, notamment ORL et pulmonaires. Ces comorbidités influent sur les possibilités thérapeutiques mais également directement sur le pronostic de ces patients. Ces derniers présentent un fort risque opératoire et sont, pour la plus part, inopérables d'emblée. Toutefois, en l'absence de métastase à distance, ils sont éligibles à un traitement de radio-chimiothérapie. L'absence de bénéfice sur la survie d'une chirurgie après une réponse complète à la radio-chimiothérapie a permis à la radio-chimiothérapie exclusive de s'imposer comme le traitement de référence pour les patients présentant une tumeur localement avancée $e^{1,2}$. La prise en charge de ces patients au CHU de Rouen consiste en une radiochimiothérapie exclusive selon le protocole d'Herskovic; la chirurgie n'intervenant plus qu'en situation de rattrapage en cas d'absence de réponse complète à la radio-chimiothérapie.

\footnotetext{
${ }^{1}$ Bedenne L, Michel P, Bouche O, Triboulet JP, Conroy T, Pezet D, et al. Randomized phase III trial in locally advanced esophageal cancer: radiochemotherapy followed by surgery versus radiochemotherapy alone (FFCD 9102). J Clin Oncol $2007 ; 25: 1160-8$

2 Stahl M, Stuschke M, Lehmann N, Meyer HJ, Walz MK, Seeber S, et al. Chemoradiation With and Without Surgery in Patients With Locally Advanced Squamous Cell Carcinoma of the Esophagus. J Clin Oncol 2005; 23: 2310-7.
} 
La TEP FDG (tomographie d'émission de positon au fluorodésoxyglucose) fait partie des examens recommandés dans le bilan d'extension du cancer de l'œsophage au côté du scanner et de l'écho-endoscopie. L'autorisation de mise sur le marché (AMM) du FDG spécifie son indication dans la stadification du cancer de l'œsophage. Le guide de bon usage des examens d'imagerie médicale, rédigé conjointement par la société française de radiologie et la société française de médecine nucléaire et d'imagerie moléculaire, fait état au $1^{\mathrm{er}}$ janvier 2013 d'un examen indiqué dans le diagnostic et le bilan d'extension du cancer de l'œsophage avec un grade de recommandation B. La TEP FDG est reconnu comme étant l'examen le plus sensible pour la détection d'une atteinte ganglionnaire ou métastatique à distance. L'examen TEP initial permet également de mieux définir le volume cible pour la radiothérapie en assurant une bien meilleure visualisation de l'extension en hauteur de la tumeur. La TEP FDG ne fait pas l'objet de recommandation précise pour l'évaluation de l'efficacité du traitement, mais elle est largement utilisée dans cette indication. L'absence de réponse complète en TEP après la radio-chimiothérapie est un facteur de mauvais pronostic clairement établi à ce jour. Toutefois, Les méta-analyses de Kwee ${ }^{3}$ et de $\mathrm{Omloo}^{4}$ n'ont pas retrouvé d'intérêt pronostique aux paramètres quantitatifs de la TEP initiale, ni à la variation des ces paramètres que ce soit au cours du traitement ou à distance de la fin du traitement.

Une étude prospective multicentrique à l'initiative du service de médecine nucléaire du Centre Henri Becquerel et du CHU de Rouen ${ }^{5}$, portant sur la recherche de facteurs prédictifs précoces de la réponse à la radio-chimiothérapie dans le cancer de l'œsophage par la réalisation d'une TEP précoce à 21 jours du début du traitement par radio-chimiothérapie, n'a pas démontré d'intérêt pronostic à la variation des paramètres quantitatifs entre la TEP initiale et précoce mais a retrouvé que le volume métabolique initial était prédictif de la réponse au traitement. Un nombre réduit d'étude évaluant la valeur pronostic du volume métabolique initial ont été réalisées jusqu'alors. Les résultats de ces études ont été synthétisés dans le tableau suivant.

\footnotetext{
${ }^{3}$ Kwee RM. Prediction of Tumor Response to Neoadjuvant Therapy in Patients with Esophageal Cancer with Methods : Results : Conclusion : Radiology. 2010;254:707-17.

4 Omloo JMT, van Heij1 M, Hoekstra OS, van Berge Henegouwen MI, van Lanschot JJB, Sloof GW. FDG-PET parameters as prognostic factor in esophageal cancer patients: a review. Ann. Surg. Oncol. 2011;18:3338-52.

5 Palie O, Michel P, Ménard J-F, Rousseau C, Rio E, Bridji B, et al. The predictive value of treatment response using FDG PET performed on day 21 of chemoradiotherapy in patients with oesophageal squamous cell carcinoma. A prospective, multicentre study (RTEP3). Eur. J. Nucl. Med. Mol. Imaging. 2013;40:1345-55.
} 


\begin{tabular}{|c|c|c|c|c|c|c|}
\hline $\begin{array}{l}\text { Auteurs } \\
\text { revue année }\end{array}$ & $\begin{array}{l}\text { Pays } \\
\text { (inclusion) }\end{array}$ & Type d'étude & $\begin{array}{c}\mathrm{n} \\
\mathrm{ADK} / \mathrm{CE}\end{array}$ & $\begin{array}{c}\text { méthode de définition } \\
\text { du volume }\end{array}$ & résultat & Test statistique \\
\hline $\begin{array}{l}\text { Mamede et al. } \\
\text { AJCO } 2007\end{array}$ & Etats-Unis & $\begin{array}{l}\text { rétrospectif } \\
\text { monocentrique }\end{array}$ & $\begin{array}{c}25 \\
22 / 3\end{array}$ & $\begin{array}{l}\text { semi automatique } \\
\text { seuil = SUVmax-(SUVmean } \\
\text { foie sain }+2 \text { DS) }\end{array}$ & prédictif du stade $T$ & $\begin{array}{c}\text { ROC } \\
(\operatorname{Se} 76,9 \%)\end{array}$ \\
\hline $\begin{array}{l}\text { Zhu et al. } \\
\text { BJR } 2012\end{array}$ & $\begin{array}{c}\text { Chine } \\
(2003-09)\end{array}$ & rétrospectif & $\begin{array}{c}41 \\
0 / 41\end{array}$ & $\begin{array}{l}\text { automatique } \\
\text { seuil à } 2,5\end{array}$ & corrélé au stade $\mathrm{N}$ & $\begin{array}{l}\text { Spearman } \\
\mathrm{p}<0,001\end{array}$ \\
\hline $\begin{array}{l}\text { Blom et al. } \\
\text { EJNM } 2012\end{array}$ & $\begin{array}{l}\text { Pays-Bas } \\
(2008-11)\end{array}$ & $\begin{array}{l}\text { rétrospectif } \\
\text { monocentrique }\end{array}$ & $\begin{array}{c}79 \\
53 / 18\end{array}$ & $\begin{array}{l}\text { automatique } \\
\text { basée sur le rapport } \\
\text { signal/bruit de fond }\end{array}$ & $\begin{array}{l}\text { prédictif de la réponse à } \\
\text { la radio-chimiothérapie }\end{array}$ & $\begin{array}{l}\text { Mann-Whitney } \\
\text { Kruskal Wallis } \\
\text { (NS) }\end{array}$ \\
\hline $\begin{array}{l}\text { Hatt et al. } \\
\text { EJNM } 2011\end{array}$ & $\begin{array}{c}\text { France } \\
(2004-08)\end{array}$ & $\begin{array}{l}\text { rétrospectif } \\
\text { monocentrique }\end{array}$ & $\begin{array}{c}50 \\
14 / 36\end{array}$ & $\begin{array}{l}\text { automatique } \\
\text { FLAB }\end{array}$ & $\begin{array}{l}\text { prédictif de la réponse à } \\
\text { la radio-chimiothérapie }\end{array}$ & $\begin{array}{c}\mathrm{ROC} \\
(\mathrm{Se}>75 \%)\end{array}$ \\
\hline $\begin{array}{l}\text { Palie et al. } \\
\text { EJNM } 2013\end{array}$ & France & $\begin{array}{l}\text { prospectif } \\
\text { multicentrique }\end{array}$ & $\begin{array}{c}48 \\
0 / 48\end{array}$ & manuelle & $\begin{array}{l}\text { prédictif de la réponse à } \\
\text { la radio-chimiothérapie }\end{array}$ & $\underset{p=0,041}{\operatorname{Cox}}$ \\
\hline $\begin{array}{l}\text { Hyun et al. } \\
\text { ASO } 2010\end{array}$ & $\begin{array}{c}\text { Corée } \\
(2000-02)\end{array}$ & $\begin{array}{l}\text { rétrospectif } \\
\text { monocentrique }\end{array}$ & $\begin{array}{c}151 \\
5 / 146\end{array}$ & $\begin{array}{l}\text { automatique } \\
3 \text { seuils fixe fonction du } \\
\text { SUVmax }\end{array}$ & $\begin{array}{l}\text { facteur pronostic de } \\
\text { survie globale }\end{array}$ & $\begin{array}{c}\text { Cox } \\
p=0,021\end{array}$ \\
\hline $\begin{array}{l}\text { Créhange et } \\
\text { al. AJCO } 2006\end{array}$ & $\begin{array}{c}\text { France } \\
(1993-01)\end{array}$ & $\begin{array}{l}\text { rétrospectif } \\
\text { monocentrique }\end{array}$ & $\begin{array}{c}148 \\
19 / 125\end{array}$ & $\begin{array}{l}\text { Manuelle (volume TDM) } \\
\text { formule intégrant } 3 \\
\text { diamètres et la hauteur }\end{array}$ & $\begin{array}{l}\text { facteur pronostic de } \\
\text { survie globale }\end{array}$ & $\begin{array}{c}\text { Cox } \\
p=0,041\end{array}$ \\
\hline $\begin{array}{l}\text { Hatt et al. } \\
\text { EJNM } 2011\end{array}$ & France & $\begin{array}{l}\text { rétrospectif } \\
\text { monocentrique }\end{array}$ & $\begin{array}{c}45 \\
12 / 33\end{array}$ & $\begin{array}{l}\text { automatique } \\
\text { FLAB }\end{array}$ & $\begin{array}{l}\text { facteur pronostic de } \\
\text { survie globale }\end{array}$ & $\begin{array}{c}\text { Cox } \\
\mathrm{p}=0,0017\end{array}$ \\
\hline
\end{tabular}

$\mathrm{n}$ : nombre de patients inclus ; ADK : adénocarcinome ; CE : cancer épidermoïde ; DS : déviation standard ; ROC : receiver operating caracteristic ; Se : sensibilité ; NS : non significatif; FLAB : Fuzzy Locally Adaptive Bayesian

La majorité de ces études ne compte qu'un faible nombre de patients. L'étude de Créhange et al. ${ }^{6}$ qui compte 125 patients atteints de cancer épidermoïdes de l'œsophage a étudié la valeur pronostic du volume défini sur la TDM et non sur la TEP. L'étude de Hyun et al. ${ }^{7}$ a été réalisée en Corée où l'épidémiologie de la maladie est particulière avec notamment une incidence élevée de la maladie et une prédominance encore plus marqué du type histologique épidermoïde ${ }^{8}$ et ces résultats doivent être confirmés en Europe.

\footnotetext{
${ }^{6}$ Créhange G, Bosset M, Lorchel F, Fabrice L, Buffet-Miny J, Dumas JL, et al. Tumor volume as outcome determinant in patients treated with chemoradiation for locally advanced esophageal cancer. Am. J. Clin. Oncol. 2006;29:583-7.

${ }^{7}$ Hyun SH, Choi JY, Shim YM, Kim K, Lee SJ, Cho YS, et al. Prognostic value of metabolic tumor volume measured by 18F-fluorodeoxyglucose positron emission tomography in patients with esophageal carcinoma. Ann. Surg. Oncol. 2010;17:115-22.

${ }^{8}$ Bashash M, Hislop TG, Shah AM, Le N, Brooks-Wilson A, Bajdik CD. The prognostic effect of ethnicity for gastric and esophageal cancer: the population-based experience in British Columbia, Canada. BMC Cancer. 2011;11:164.
} 
La TEP est disponible à Rouen depuis la fin de l'année 2004. L'ensemble des patients présentant un cancer épidermoïde de l'œsophage localement avancé pris en charge au CHU de Rouen a été adressé pour un examen TEP initial dans le cadre du bilan d'extension dès la mise à disposition de cette technique dans la région. Tous les examens ont été réalisés sur le même appareil, au centre Henri Becquerel. Le service de médecine nucléaire du centre Henri Becquerel est resté l'unique service de la région à disposer de la TEP jusqu'en 2011, garantissant une série consécutive de patients et une homogénéité des examens réalisés. La plupart de ces patients ont également fait l'objet d'un suivi en TEP pour l'évaluation de la réponse et lors de la suspicion d'une récidive.

Fort des résultats de l'étude RTEP3 et d'une base de données conséquente et de qualité, nous avons voulu préciser l'intérêt pronostique du volume métabolique initial dans une population spécifique de patients porteurs de cancer épidermoïde de l'œsophage localement avancé, et dépasser le critère pronostique intermédiaire qu'est la réponse au traitement pour atteindre des critères finalistes dont la signification clinique est plus forte comme la survie sans maladie ou la survie globale. 
L'article original présenté ci-après est soumis pour publication à l'European Journal of Nuclear Medicine and Molecular Imaging (Impact Factor 2012 : 5,114).

Ce travail fera également l'objet d'une présentation affichée au 52ème colloque de Médecine Nucléaire de langue française qui se tiendra à Saint-Étienne du 16 au 19 mai 2014.

Le résumé de ce travail a par ailleurs été soumis au congrès annuel de la Society of Nuclear Medicine and Molecular Imaging qui se tiendra du 7 au 11 juin 2014 à Saint Louis, Missouri, ainsi qu'aux 7èmes journées scientifiques du Cancéropôle nord-ouest qui se tiendront à Deauville du 14 au 16 mai 2014. 


\title{
Pretreatment metabolic tumour volume is predictive of disease-free survival and overall survival in patients with oesophageal squamous cell carcinoma.
}

\author{
Charles Lemarignier ${ }^{1}$ \\ Frédéric Di Fiore ${ }^{2,3}$ \\ Charline Marre $^{2}$ \\ Sébastien Hapdey ${ }^{1,4}$ \\ Romain Modzelewski ${ }^{1,4}$ \\ Pierrick Gouel $^{1}$

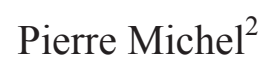 \\ Bernard Dubray ${ }^{4,5}$ \\ Pierre Vera ${ }^{1,4}$
}

1. Department of Nuclear Medicine, Centre Henri Becquerel and Rouen University Hospital, Rouen, France

2. Department of Gastroenterology Rouen University Hospital, Rouen, France

3. Department of Medical oncology, Centre Henri Becquerel, Rouen, France

4. QuantIF (Litis EA 4108) University of Rouen, Rouen, France

5. Department of Radiotherapy and Medical Physics, Centre Henri Becquerel and Rouen University Hospital, Rouen, France

\section{Keywords}

Quantitative metabolic parameters - metabolic tumour volume

Outcome

Oesophageal carcinoma

Chemo radiotherapy

PET-CT 


\section{Abstract}

\section{Purpose}

It has been suggested that FDG PET has predictive value for the prognosis of treated oesophageal carcinoma. However, the studies reported in the literature have shown discordant results. The aim of this study was to determine whether pre-therapy quantitative metabolic parameters correlate with patient outcomes.

\section{Methods}

Sixty-seven patients with a histological diagnosis of oesophageal squamous cell carcinoma were included in this study. Each patient underwent FDG PET (4.5 MBq/kg) before chemo radiotherapy, following Herskovic's protocol. The quantitative analysis was performed using the following parameters: age, weight loss, location, N stage, WHO performance status, $\mathrm{MTV}_{\mathrm{p}}$ and $\mathrm{MTV}_{\mathrm{p}}$, (metabolic tumour volume determined by two different physicians), $\mathrm{MTV}_{40 \%}$ (volume for a threshold of $40 \%$ of SUVmax), MTV (volume automatically determined with a contrast-based adaptive threshold method), SUVmax, SUVmean and TLG.

\section{Results}

The $\mathrm{MTV}_{\mathrm{p}}$ and $\mathrm{MTV}_{40 \%}$ were highly correlated (Pearson's index 0.92). The SUVmean $\mathrm{p}$ and SUVmean $_{40 \%}$ were also correlated (Pearson's index 0.86). The $\mathrm{TLG}_{\mathrm{p}}$ and $\mathrm{TLG}_{40 \%}$ were correlated (Pearson's index 0.98) as well. Similarly, the parameters obtained with the adaptive threshold method $\left(\mathrm{MTV}_{\mathrm{a}}, \mathrm{SUVmean}\right.$ and $\left.\mathrm{TLG}_{\mathrm{a}}\right)$ correlated with those obtained manually $\left(\mathrm{MTV}_{\mathrm{p}}, \mathrm{SUVmean}_{\mathrm{p}}\right.$ and $\left.\mathrm{TLG}_{\mathrm{p}}\right)$. The manual metabolic tumour volume determination $\left(\mathrm{MTV}_{\mathrm{p}}\right.$ and $\mathrm{MTV}_{\mathrm{p}}$ ) was reproducible. The multivariate analysis for disease-free survival (DFS) showed that a larger $\mathrm{MTV}_{\mathrm{p}}$ was associated with a shortened DFS $(\mathrm{p}=0.004)$ and that a high SUVmax was associated with an increased DFS $(p=0.02)$. The multivariate analysis for overall survival (OS) showed that a larger $\mathrm{MTV}_{\mathrm{p}}$ was associated with a shortened OS $(p=0.01)$ and that a tumour that had developed in the distal oesophagus was associated with an increased $\operatorname{OS}(\mathrm{p}=0.005)$. The other parameters were not statistically significant.

\section{Conclusion}

Metabolic tumour volume is a major prognostic factor for DFS and OS in patients with oesophageal squamous cell carcinoma. High SUVmax values are paradoxically associated with a longer survival. The location of the tumour also appears to affect the prognosis. 


\section{Introduction}

Oesophageal carcinoma affects more than 450,000 people worldwide, with a predominant histologic type of squamous cell carcinoma [1]. The prognosis remains poor, with an estimated five-year survival barely higher than 15\% [2]. In 2010, 4,240 new cases of oesophageal carcinoma and 3,470 deaths related to oesophageal carcinomas were registered in France [3]. In the majority of cases, patients present with a locally advanced oesophageal carcinoma which is defined as a tumour with oesophageal tissue involvement, or contiguous structure invasion or lymph node involvement [4]. In these patients, definitive chemo radiotherapy has become a first-line therapeutic option [5]. Considering the potentially severe side effects of the treatment modalities, accurate staging is essential to determine the relevant strategy for each patient. A method that can reliably predict treated patients' survival would be recommended to appropriately select the patients who will benefit from the treatment and thus avoid unnecessary treatment.

Fluoro-2-deoxy-glucose positron emission tomography (FDG PET) is already well established for the staging of oesophageal cancer, with a reasonable sensitivity and specificity in the detection of distant metastases [6]. It has a higher diagnostic performance than CT [7]. FDG PET alters the management of patients because it allows more accurate staging [8], but it remains unable to predict treated patients outcomes. Indeed, the comparison between the initial PET and the post-treatment PET has shown either non-significant results [8] or discordant results and did not appear to have any therapeutic consequences [9-11]. In addition, the appropriate timing of the evaluation of the treatment response (three to four weeks after the last cycle of chemotherapy and at least two months after radiotherapy) is still debated [12]. Post-treatment evaluation has thus been considered of low predictive potential, which has increased the interest in per treatment evaluation. A meta-analysis studying the modification of the PET parameters between the initial PET and a PET performed after the beginning of the treatment failed to show significant results [13]. More specifically, early evaluation during treatment yielded discordant results $[14,15]$. Similarly, the per treatment evaluation has not shown its predictive potential yet. Therefore, we concluded that it would be beneficial to the patient and more cost-effective to be able to predict therapy response and survival from a single baseline PET acquired before the initiation of the treatment. Recently, an increasing number of studies have focused on evaluating only the initial PET parameters and concentrating on the volume parameters or the combined volume and SUV parameters 
rather than on SUV parameters alone, as has been the case for many years [10,16-20]. Nevertheless, these studies were based on small series of patients or heterogeneous populations in term of histological types (including both adenocarcinomas and squamous cell carcinomas) and treatment modalities (exclusive surgery, adjuvant therapy, neoadjuvant therapy, exclusive chemo radiotherapy, exclusive chemotherapy) and used surrogate endpoints, such as treatment response, rather than survival.

The aim of our study was to determine whether the quantitative metabolic parameters of the initial PET, such as the metabolic tumour volume (MTV), could be predictive of the outcomes in patients with oesophageal squamous cell carcinoma treated with chemo radiotherapy. 


\section{Materials and methods}

\section{Study design}

This study was a single-arm, retrospective, single-institution clinical trial. We included all patients with oesophageal squamous cell carcinoma who were eligible for exclusive chemo radiotherapy (CRT) and in whom a diagnostic PET CT had been performed. Patients with distant visceral metastases were excluded. The flow chart is shown in the figure below.

Figure 1 Flow-chart

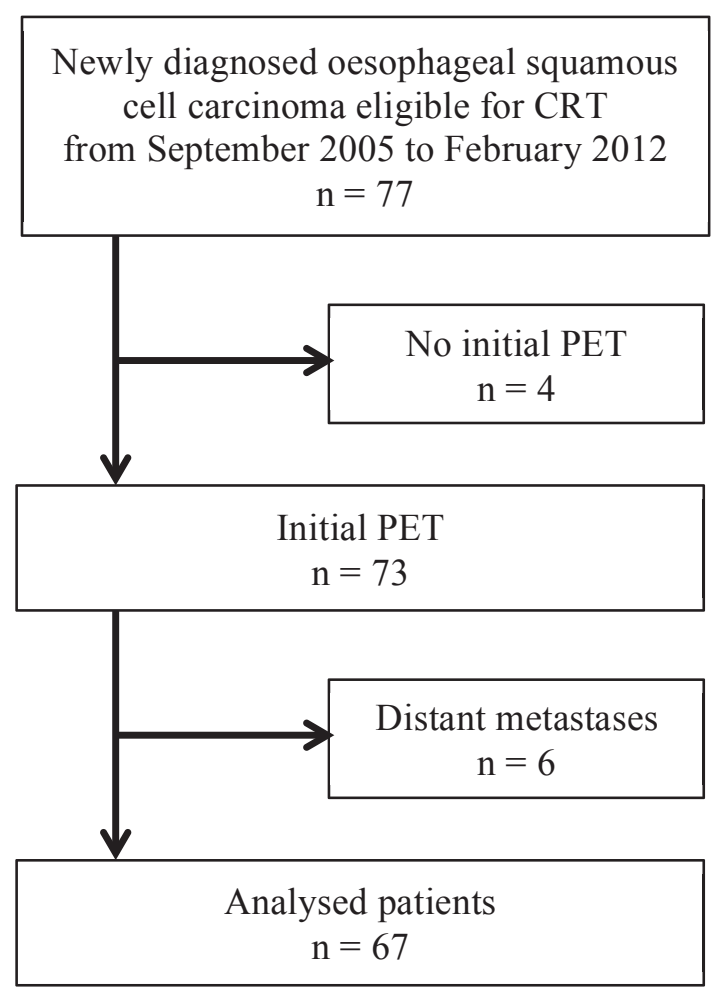

From September 2005 to March 2012, 77 patients with an advanced stage oesophageal squamous cell carcinoma who were eligible for exclusive chemo radiotherapy were newly diagnosed in our centre. Four of the 77 patients with a squamous cell carcinoma did not have an initial PET and in six patients, the PET confirmed or revealed distant metastases. Sixtyseven consecutive patients were thus included in the study.

The PET must be performed prior to any treatment. The squamous cell carcinoma was pathologically proven with a biopsy obtained during the diagnostic oesophagoscopy. The initial tumour staging and location were based on an oesophagoscopy, with biopsies, endoscopic ultrasonography, chest and abdominal CT and FDG PET. Staging was performed 
according to the $6^{\text {th }}$ edition (2002) of the TNM classification of the American Joint Committee on Cancer (AJCC) and the Union for International Cancer Control (UICC). The following data were recorded for each patient: age, sex, World Health Organization performance status (WHO), weight loss from baseline, dysphagia grade and tumour location. Dysphagia was assessed using the Dysphagia Grading System:

Grade 0: able to swallow all solid foods without difficulty,

Grade1: able to swallow solid foods with some difficulty,

Grade 2: able to swallow soft or semi-solid foods only,

Grade 3: able to swallow liquefied foods and liquids only,

Grade 4: unable to swallow liquids/saliva.

The demographic and clinical characteristics of the patients are listed in the Table below.

Table 1 Demographic and clinical characteristics of the 67 patients

Demographic

$\begin{array}{ll}\begin{array}{l}\text { Age, years } \\ \text { median (range) } \\ \text { Sex }\end{array} & 63(46-86) \\ \quad \text { Male } & 53(79 \%) \\ \quad \text { Female } & 14(21 \%)\end{array}$

\section{Clinical}

WHO

$\begin{array}{rr}0 & 33(49 \%) \\ 1 & 29(43 \%) \\ 2 & 5(8 \%)\end{array}$

Weight loss

$\begin{array}{ll}<10 \% & 44(66 \%) \\ \geq 10 \% & 23(34 \%)\end{array}$

Dysphagia

$\begin{array}{lr}0 & 4(6 \%) \\ 1 & 29(43 \%) \\ 2 & 26(39 \%) \\ 3 & 7(10 \%) \\ 4 & 1(2 \%)\end{array}$


All of the patients were treated with chemo radiotherapy according to the Herskovic's scheme [21]. This included external radiotherapy delivered by a two/three-field technique at 2 Gy per fraction per day and five sessions per week for a total of 50 Gy over five weeks. IntensityModulated Radio Therapy (IMRT) was not used. All of the doses were calculated without heterogeneity correction. The Gross Tumour Volume (GTV) was determined using the metabolic tumour volume delineated by the nuclear physician on the initial TEP. To account for microscopic disease extension, a Clinical Target Volume (CTV) was defined by adding to the GTV a $5 \mathrm{~cm}$ cranio-caudal margin. The GTV and all clinically suspect lymph nodes were to receive $50 \mathrm{~Gy}$. The CTV and non-suspect lymph nodes were to receive $40 \mathrm{~Gy}$. This was combined with chemotherapy consisting of two cycles of cisplatin $\left(75 \mathrm{mg} / \mathrm{m}^{2}\right)$ and 5 fluorouracil (four days of continuous infusion of $1,000 \mathrm{mg} / \mathrm{m}^{2}$ ) in weeks 1 and 5 .

The response assessment included a clinical examination, CT, FDG PET and oesophagoscopy with biopsies. These assessments were performed one month after the end of the treatment. The patients were classified as showing a clinical complete response (CR) to chemo radiotherapy if no residual tumour was detected on endoscopy (negative biopsies) and if no occurrence of loco regional or distant disease was identified either on CT evaluation or FDG PET evaluation. The patients were classified as showing a non-complete response (nCR) when a residual tumour, loco regional or distant disease was detected or when patient death occurred. Electronic patient files were available for gathering the clinical data of patients retrospectively and for long-term follow-up. Twelve patients in our study were operated on: six for a non-complete response, five for a recurrence (four recurrences in the first year following treatment evaluation) and one who underwent surgery although he had achieved a complete response. The initial characteristics, DFS and OS of these 12 operated patients were not significantly different from the characteristics of our entire cohort. The tumour characteristics, outcomes and follow-up are summarised in the following table. 
Table 2 Tumour characteristics, outcome and follow-up

\begin{tabular}{|c|c|}
\hline \multicolumn{2}{|l|}{ Tumour location } \\
\hline Upper third & $16(24 \%)$ \\
\hline Middle third & $32(48 \%)$ \\
\hline Lower third & $19(28 \%)$ \\
\hline \multicolumn{2}{|l|}{ T stage } \\
\hline $\mathrm{Tx}$ & $4(6 \%)$ \\
\hline $\mathrm{T} 2$ & $8(12 \%)$ \\
\hline $\mathrm{T} 3$ & $51(76 \%)$ \\
\hline $\mathrm{T} 4$ & $4(6 \%)$ \\
\hline \multicolumn{2}{|l|}{$\mathrm{N}$ stage } \\
\hline N0 & $17(25 \%)$ \\
\hline N1 & $50(75 \%)$ \\
\hline \multicolumn{2}{|l|}{ M stage } \\
\hline M0 & $59(88 \%)$ \\
\hline M1a & $8(12 \%)$ \\
\hline \multicolumn{2}{|l|}{ AJCC stage } \\
\hline $\mathrm{x}$ & $4(6 \%)$ \\
\hline IIA & $16(24 \%)$ \\
\hline IIB & $4(6 \%)$ \\
\hline III & $35(52 \%)$ \\
\hline IVA & $8(12 \%)$ \\
\hline Complete response & $50(75 \%)$ \\
\hline Relapse & $26(39 \%)$ \\
\hline Non-complete response & $17(25 \%)$ \\
\hline Death & $43(64 \%)$ \\
\hline related to $\mathrm{OC}$ & $32(48 \%)$ \\
\hline 1 year survival & $51(76 \%)$ \\
\hline $\begin{array}{l}\text { follow-up, month } \\
\text { median (range) }\end{array}$ & $47(15-81)$ \\
\hline
\end{tabular}

\section{F-FDG PET acquisitions}

The patients were asked to fast for at least $6 \mathrm{~h}$ before the PET examination. The patients' blood glucose levels were measured. A dose of $4.5 \mathrm{MBq}$ per kilogram of 18F-FDG was injected intravenously 60 min prior to PET CT scanning. The imaging was performed with the Biograph 16 integrated PET CT scanner (Biograph Sensation 16; Siemens, Knoxville, TN, USA), which includes a 16-slice CT component and a PET system with lutetium oxyorthosilicate (LSO) crystals. For the PET imaging, the emission data were acquired from 
the base of the skull to the proximal thigh with 3 to $3.5 \mathrm{~min}$ of acquisition per bed position, depending on the patient's body mass index (BMI), each covering $16.2 \mathrm{~cm}$, at an axial sampling thickness of $2 \mathrm{~mm}$ per slice. The CT scan parameters were set to $100-120 \mathrm{kVp}$ and 100-150 mAs (based on the patient's BMI) using dose reduction software (CareDose, Siemens Medical Solutions, Hoffman Estates, Knoxville, TN, USA). Both the PET scans and the CT scans were obtained during normal tidal breathing. The PET images were reconstructed with CT-derived attenuation correction using an attenuation-weighted orderedsubset expectation maximisation (AW-OSEM) algorithm. The attenuation-corrected PET images, CT images and fused PET-CT images were available for interpretation in the axial, coronal and sagittal planes, as was a cine display of the maximum intensity projections of the PET data, using the TrueD clinical review software (Siemens MMWP workstation).

\section{PET image analysis}

The volumetric regions of interest (ROI) around the outline of the primary tumour and the nodes were placed on the axial PET images. The edges of the ROI were adjusted manually with 3D reconstructions to avoid overlap with the adjacent FDG avid structures. A threshold was selected by the visual inspection of the lesion's outline to best fit the tumour and to determine the tumour volume $\left(\mathrm{MTV}_{\mathrm{p}}\right)$. This operation was repeated by a second physician on 26 randomly selected patients in our study $\left(\mathrm{MTV}_{\mathrm{p}}\right)$ to evaluate the reproducibility. The tumour volume was also delineated with a threshold of $40 \%$ of the SUVmax $\left(\mathrm{MTV}_{40 \%}\right)$. The following parameters were collected: SUVmax, $\mathrm{SUVmean}_{\mathrm{p}}$ defined as the mean SUV within $\mathrm{MTV}_{\mathrm{p}}$, SUVmean $40 \%$ defined as the mean SUV within $\mathrm{MTV}_{40 \%}$, and the total lesion glycolysis: $\mathrm{TLG}_{\mathrm{p}}=\mathrm{SUVmean}_{\mathrm{p}} \times \mathrm{MTV}_{\mathrm{p}}$ and $\mathrm{TLG}_{40 \%}=\mathrm{SUVmean}_{40 \%} \times \mathrm{MTV}_{40 \%}$. We also used a contrast-based adaptive threshold method on an Oncoplanet Workstation (Dosisoft, Villejuif, France) to determine the MTV, SUVmean $_{\mathrm{a}}$ and $\mathrm{TLG}_{\mathrm{a}}$ [22].

\section{Statistics}

The quantitative analysis was performed using the following PET parameters: SUVmax, SUVmean $_{\mathrm{p}}, \mathrm{SUVmean}_{40 \%}, \mathrm{MTV}_{\mathrm{p}}, \mathrm{MTV}_{\mathrm{p}}, \mathrm{MTV}_{40 \%}, \mathrm{TLG}_{\mathrm{p}}, \mathrm{TLG}_{40 \%}, \mathrm{MTV}_{\mathrm{a}}, \mathrm{SUVmean}_{\mathrm{a}}$ and $\mathrm{TLG}_{\mathrm{a}}$. The means and the standard deviations were used for the descriptive statistics. When the statistical tests required a normal distribution, we used log-transformed values of the variables. The disease-free survival (DFS) time was calculated as the time from the initiation of chemo radiotherapy to the date of tumour recurrence, to the death of a patient who never had a recurrence or to the time of the treatment response evaluation for patients whose 
tumours did not respond to the treatment. The Multivariate analysis was performed using The Cox regression model. 


\section{Results}

All patients except one had an enhanced tumour uptake of the tracer; thus, a metabolic tumour volume was able to be delineated for 66 of the patients. Examples of the delineations are shown in the figure below.

\section{Figure 2}
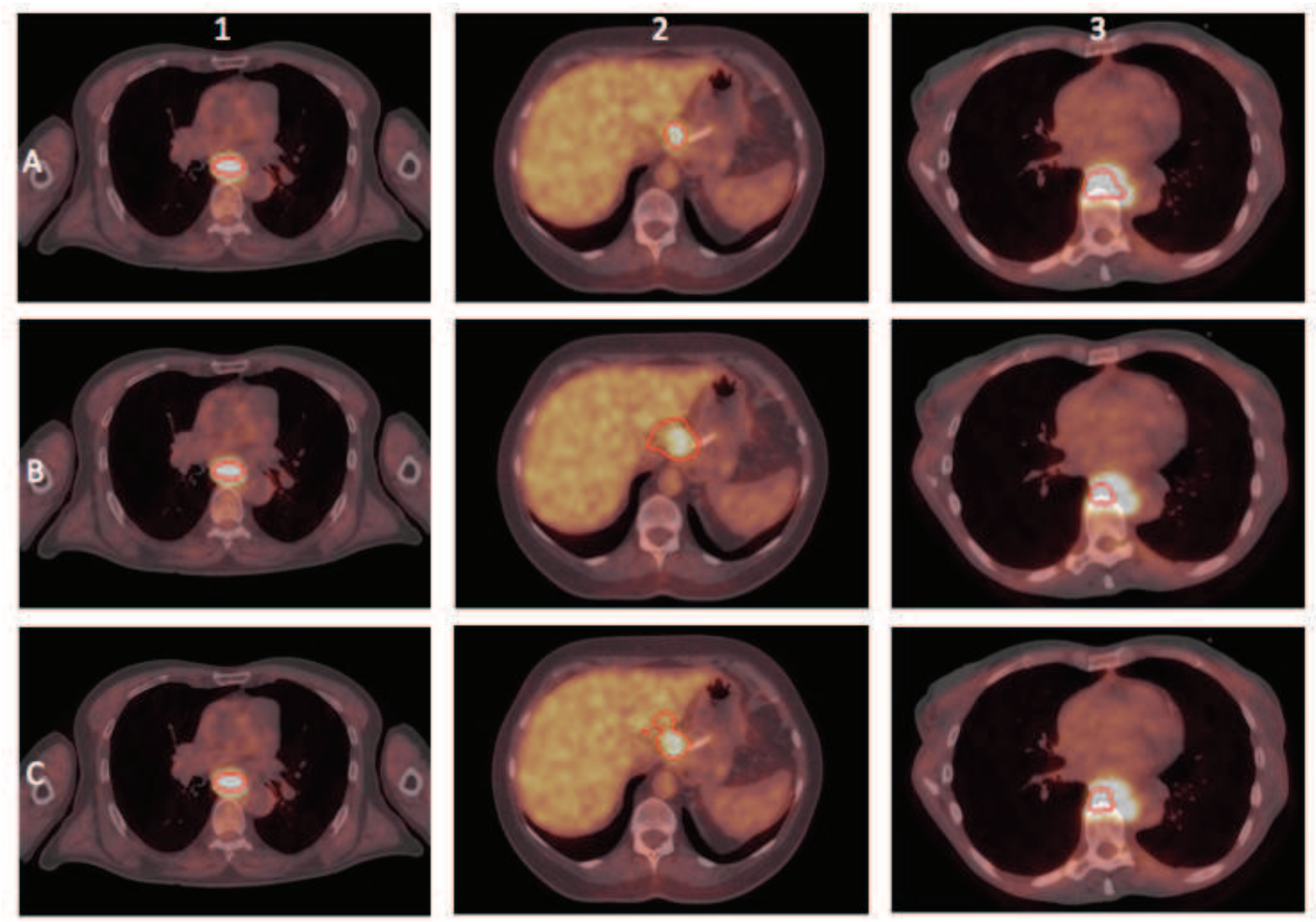

1. Example of a case in which each segmentation method gave similar results. A: physician delineation $\mathrm{MTV}_{\mathrm{p}}=18.4 \mathrm{~cm}^{3}$. B: delineation with a threshold of $40 \%$ of the SUVmax $\mathrm{MTV}_{40 \%}=17 \mathrm{~cm}^{3} . \mathrm{C}$ : delineation with the adaptive threshold method $\mathrm{MTV}_{\mathrm{a}}=19,2 \mathrm{~cm}^{3}$

2. Example of a case in which the segmentation with a threshold of $40 \%$ of the SUVmax and the segmentation with the adaptive threshold method gave larger volumes than the physician delineation, clearly overlapping on the liver. A: $M_{\mathrm{p}}=5 \mathrm{~cm}^{3}$. B: $\mathrm{MTV}_{40 \%}=31.6 \mathrm{~cm}^{3}$. C: $\mathrm{MTV}_{\mathrm{a}}=20.1 \mathrm{~cm}^{3}$

3. Example of a case in which the volume delineated by the physician was larger than the volumes defined either by the $40 \%$ of the SUVmax method or the adaptive threshold method. A: $\operatorname{MTV}_{\mathrm{p}}=22.2 \mathrm{~cm}^{3}$. B: $\operatorname{MTV}_{40 \%}=15.1 \mathrm{~cm}^{3} . \mathrm{C}: \mathrm{MTV}_{\mathrm{a}}=12.7 \mathrm{~cm}^{3}$ 
The parameters derived from the initial PET are presented in the table below.

Table 3 Initial PET derived parameters: mean \pm SD

Available for 66 patients

$\begin{array}{lr}\text { MTV }_{\mathrm{p}} & 19.5+20.7 \\ \text { MTV }_{40 \%} & 24 \pm 23.9 \\ \text { MTV }_{\mathrm{a}} & 22.9 \pm 24,9\end{array}$

Available for 65 patients

SUVmax

$13.5 \pm 5.3$

SUVmean $_{\mathrm{p}}$

$8.4 \pm 3.2$

SUVmean $_{40 \%}$

$8.2 \pm 3.5$

SUVmean $_{\mathrm{a}}$

$8.1 \pm 3.2$

$\begin{array}{ll}\mathrm{TLG}_{\mathrm{p}} & 191 \pm 253 \\ \mathrm{TLG}_{40 \%} & 217 \pm 268 \\ \mathrm{TLG}_{\mathrm{a}} & 207 \pm 260\end{array}$

Available for 26 patients $\mathrm{MTV}_{\mathrm{p}}$ $25.6 \pm 27.5$

The mean $\mathrm{MTV}_{\mathrm{p}}$ was $19.5 \mathrm{~cm}^{3}$ with a standard deviation (SD) of 20.7. The threshold for the manual delineation varied from $21 \%$ to $82 \%$ of the SUVmax, with a mean threshold of $47 \%$. The mean $\mathrm{MTV}_{40 \%}$ was $24 \mathrm{~cm}^{3}$ (SD 23.9). The SUV quantification was unavailable for one patient due to a technical problem, although we were able to delineate the tumour's volume. Consequently, we analysed the SUV parameters (SUVmax, SUVmean and TLG) of 65 of the 66 patients for whom we had delineated a tumour volume. The mean SUVmax was 13.5 (SD 5.3). The mean SUVmean pas $_{\text {p }} 8.4$ (SD 3.2) and the mean SUVmean $_{40 \%}$ was 8.2 (SD 3.5). The mean $\mathrm{TLG}_{\mathrm{p}}$ was 191 (SD 253) and the mean $\mathrm{TLG}_{40 \%}$ was 217 (SD 268). The mean MTV was $22.9 \mathrm{~cm}^{3}$ (SD 24.9) and the mean TLG 207 (SD 260). The mean MTV 2 , was 25.6 $\mathrm{cm}^{3}$ (SD 27.5) when the manual segmentation was repeated by a second physician (26 patients).

The $\mathrm{MTV}_{\mathrm{p}}$ and the $\mathrm{MTV}_{40 \%}$ were highly correlated, with a Pearson's index of 0.92 . The $\mathrm{SUVmean}_{\mathrm{p}}$ and the $\mathrm{SUVmean}_{40 \%}$ were also correlated (Pearson's index 0.86), as well as the 
$\mathrm{TLG}_{\mathrm{p}}$ and the $\mathrm{TLG}_{40 \%}$ (Pearson's index 0.98). The $\mathrm{MTV}_{\mathrm{p}}$ and the $\mathrm{MTV}_{\mathrm{a}}$ were correlated

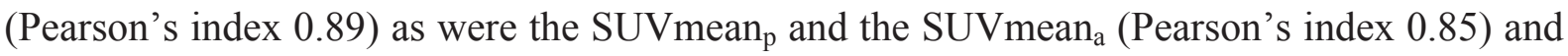
the $\mathrm{TLG}_{\mathrm{p}}$ and the $\mathrm{TLG}_{\mathrm{a}}$ (Pearson's index 0.93). The $\mathrm{MTV}_{\mathrm{p}}$ and the $\mathrm{MTV}_{\mathrm{p}}$, were highly correlated (Pearson's index 0.95) with a mean difference of $3.33 \mathrm{~cm}^{3}$. Finally, the SUVmax and $\mathrm{MTV}_{\mathrm{p}}$ were moderately correlated (Pearson's index 0.65 ).

Regarding disease-free survival, a multivariate analysis including age, weight loss, location, $\mathrm{N}$ stage, WHO performance status, $\mathrm{MTV}_{\mathrm{p}}$ and SUVmax showed that a larger volume was associated with a shortened DFS $(\mathrm{p}=0.004)$ and that a high SUVmax was associated with an increased DFS ( $p=0.02)$. The other variables were not significantly related to outcome. As for overall survival, a multivariate analysis including age, weight loss, location, WHO performance status, $\mathrm{MTV}_{\mathrm{p}}$ and SUVmax indicated that a larger volume was associated with a shortened overall survival $(\mathrm{p}=0.01)$ and that a tumour that developed from the distal oesophagus was associated with an increased overall survival $(\mathrm{p}=0.005)$. The other variables were not statistically significant. These results are reported in the table below.

Table 4 Multivariate analysis for DFS and OS

$$
\text { variables } \quad \mathrm{RR} \quad 95 \% \mathrm{CI} \quad \mathrm{p}
$$

Disease-free survival ${ }^{1}$

$\begin{array}{llll}\mathrm{MTV}_{\mathrm{p}} & 1.02 & 1.01-1.04 & 0.004 \\ \mathrm{SUVmax} & 0.93 & 0.87-0.99 & 0.02\end{array}$

Overall survival $\left.\right|^{2}$

$\begin{array}{llll}\mathrm{MTV}_{\mathrm{p}} & 1.02 & 1.004-1.04 & 0.01 \\ \text { Lower third } & 0.26 & 0.10-0.66 & 0.005\end{array}$

RR: relative risk, 95\% CI: 95\% confidence interval

${ }^{1}$ age, weight loss, location, $\mathrm{N}$ stage and WHO performance status were not statistically significant

${ }^{2}$ age, weight loss, N stage, WHO performance status and SUVmax were not statistically significant 
The Kaplan-Meier survival curves for DFS and OS are shown in the figure below.

Figure 3 Kaplan-Meier survival curves

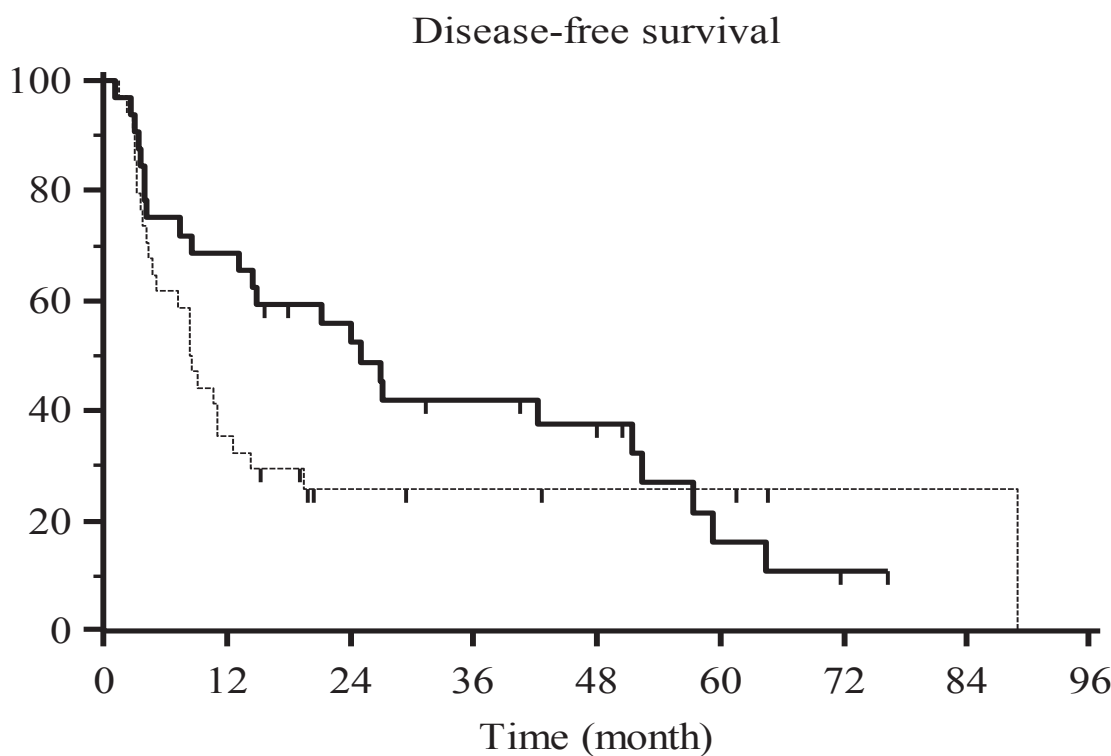

MTV

$-<14 \mathrm{ml}$ $>14 \mathrm{ml}$

Number at risk

Group: $<14 \mathrm{ml}$

$\begin{array}{lllllllll}32 & 22 & 16 & 11 & 8 & 3 & 1 & 0 & 0\end{array}$

$\begin{array}{ccccccccc}\text { Group: }>14 \mathrm{ml} \\ 34 & 12 & 5 & 4 & 3 & 3 & 1 & 1 & 0\end{array}$

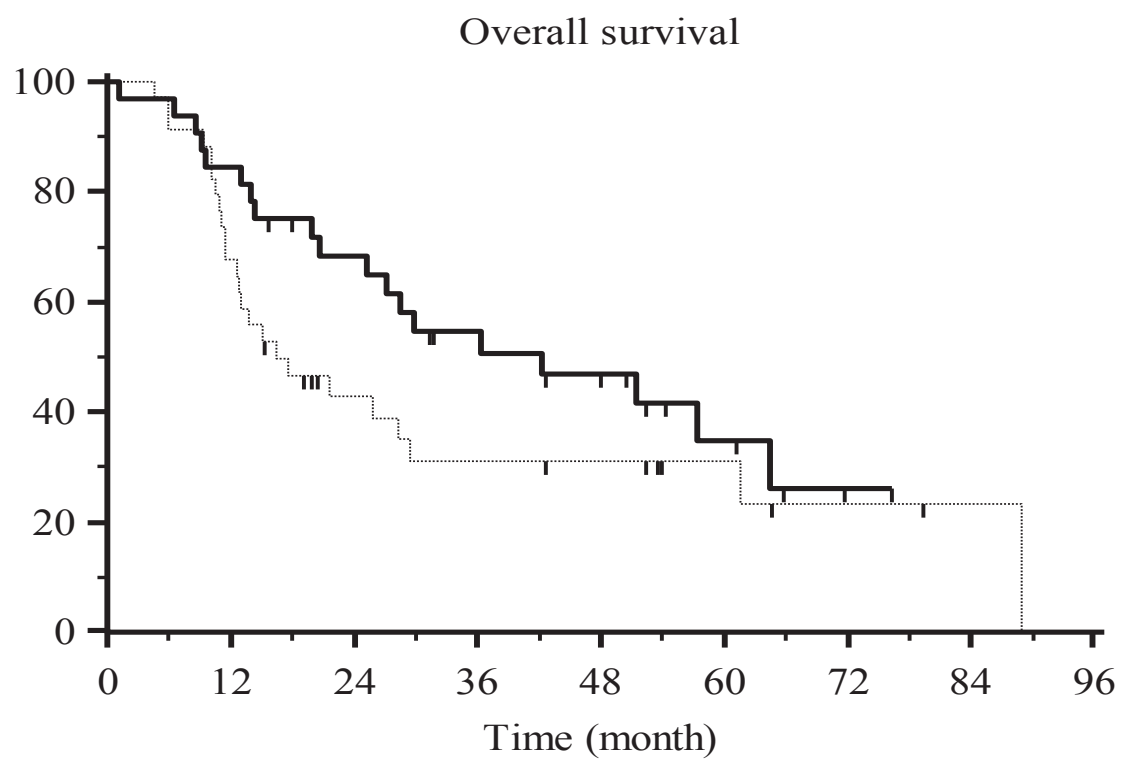

Number at risk

Group: $<14 \mathrm{~mL}$

$\begin{array}{ccccccccc}32 & 27 & 20 & 14 & 10 & 5 & 1 & 0 & 0 \\ \text { Group: }>14 \mathrm{~mL} & & & & & & & & \\ 34 & 23 & 11 & 8 & 7 & 4 & 2 & 1 & 0\end{array}$

MTV

$<14 \mathrm{~mL}$ $>14 \mathrm{~mL}$ 
The patients were divided into two groups using the median $\operatorname{MTV}_{\mathrm{p}}\left(14 \mathrm{~cm}^{3}\right)$ as a threshold. Two years after beginning the treatment, more than $50 \%$ of the patients with an MTV below the median were still in complete remission, whereas more than $80 \%$ of the patients with an MTV above the median never had a response to the treatment, had a recurrence or died. More than $60 \%$ of the patients from the group with an MTV below the median were still alive at two years, in contrast with less than $40 \%$ of the patients with an MTV above the median. The median overall survival was 42 months in patients with an MTV below $14 \mathrm{~cm}^{3}$ and 16 months in patients with an MTV above $14 \mathrm{~cm}^{3}$. The median disease-free survival was 25 months in patients with an MTV below $14 \mathrm{~cm}^{3}$ and eight months in patients with an MTV above $14 \mathrm{~cm}^{3}$. 


\section{Discussion}

Based on one of the largest series of patients suffering from oesophageal cancer and including only those in whom advanced stage squamous cell carcinoma was treated with exclusive chemo radiotherapy, our study found that the MTV manually defined on the initial PET was an independent prognostic factor of disease-free survival and overall survival. Our study also found that a high SUVmax was linked to an increased DFS.

We decided to study the parameters from the initial extension PET because the earlier a potential change in the therapeutic scheme is determined, the greater the impact will be. This initial evaluation would induce possible changes earlier than a classical treatment evaluation would, whether it is performed at the end of the treatment or even during the treatment $[9,10]$. The single initial PET evaluation also enabled us to avoid two issues: oesophagitis, rapidly appearing during the treatment and making PET interpretation much more difficult [23], and the precise time during or after the treatment when the evaluation should be performed, which is still an unresolved issue. Furthermore, the methods for comparing the sequential examinations for a given patient have not been clearly established yet. For example, the PERCIST criteria are very restrictive in terms of examination reproducibility. One-third of patients exceeds the limits of variation in the SULmean in a non-diseased liver to meet the inclusion criteria for the therapy response evaluation as required by the PERCIST protocol [24], making this method very difficult to use in clinical practice. Finally, the prognostic prediction of a single PET is more cost-effective than any sequential evaluation on an extra examination.

We did not assess the treatment response on resected oesophageal tumours but rather on the biopsies in consideration of the therapeutic scheme our patients typically undergo. The evaluation of a resected tumour is generally considered to be the gold standard. However, its use would have artificially modified the outcome, as those patients do not usually undergo surgery. Indeed, the patients included in our study presented initially with unresectable tumours; if the assessment after chemo radiotherapy showed a clinically complete tumour response, we could avoid surgery, and if the assessment showed a non-complete response, a salvage surgery could be discussed. Thus, very few of our patients with initially unresectable tumours actually underwent surgery. The initial characteristics, DFS and OS of the 12 operated patients were not significantly different from the characteristics of our entire cohort. 
We favoured simple methods that are reproducible with tools available on any routine review station. Our manual delineation takes only a few minutes and does not significantly extend the duration of the examination's interpretation. The manual delineation may be considered to be reproducible, as the volumes delineated by the two different physicians were highly correlated with a mean difference of $3.33 \mathrm{~cm}^{3}$. Prior to this study, the two physicians together delineated 10 cases as a training course until a consensus was achieved. These 10 cases were not part of the study's population.

The present study found that metabolic tumour volume $\left(\mathrm{MTV}_{\mathrm{p}}\right)$ is the best predictive factor for both disease-free survival and overall survival in a multivariate analysis. This result is consistent with previous studies [10]. Regarding treatment response, Blom et al. observed a non-significant correlation $(n=79)$ [16], Hatt et al. found that MTV was predictive of treatment response with a sensitivity over $75 \%(n=50)$ [19], and Palie et al. demonstrated that MTV was predictive of treatment response $(n=48)$ [14]. Regarding overall survival, Hyun et al. found, in a retrospective monocentric study on a Korean population with epidemiologic specificity, that MTV was an independent predictive factor $(n=151)$ [17], Créhange et al. had already established that the tumour volume determined on CT-scan images was an independent predictive factor $(\mathrm{n}=148)$ [25], and Hatt et al. demonstrated the predictive value of MTV determined automatically by the FLAB method $(n=45)$ [26]. Our study confirms and extends these previous results.

Our study also found that high SUVmax values are paradoxically associated with a longer disease-free survival in a multivariate analysis. Many studies report that the prognostic value of the initial SUVmax has not been demonstrated $[9,10,12]$. Specifically, for Downey et al., the initial SUVmax was not a prognostic factor [8]; for Wieder et al. it was not predictive for treatment response [15] and for Hatt et al. it was not predictive for overall survival [26]. However, at the same time, some studies report that the decrease in the SUVmax on a later examination was associated with response, DFS and OS [9,10,27], even though Downey et al. and a review of the literature did not confirm this result $[8,13]$. Additionally, in the previously cited study of Levine et al. [27], high SUVmax values on the initial PET were associated with a complete histological response and low values were associated with a non-complete response, which our results for DFS confirm.

Finally, our study found in a multivariate analysis that the patients with a tumour that had developed from the distal oesophagus had a longer overall survival than the patients with a 
tumour that had developed from the upper or the middle oesophagus. Studies have shown that an inferior location is associated with a better prognosis [28-30], although this result appears to be variable depending on ethnicity [31], and that some studies also report conflicting results [32]. However, the results reported in the two previously mentioned studies must be taken with caution because they grouped the two primary histological types (i.e., adenocarcinoma and squamous cell carcinoma) and also merged locations, for example, gastric cardia with the lower third of the oesophagus, which may not necessarily have the same prognosis. This better prognosis for tumours originating from the distal oesophagus has been suspected to be a result of the increased resectability of such tumours. Indeed, in our study, seven patients of the 19 with a tumour in the lower third of the oesophagus were operated on compared with only five of the 48 patients with tumours in the upper or the middle third of the oesophagus. In our study, surgery may have modified the patients' outcomes and led to a possible overestimation of the location's effect.

The $\mathrm{MTV}_{40 \%}$ did not have a better prognostic value in our multivariate analysis than the $\mathrm{MTV}_{\mathrm{p}}$. The determination of a tumour's volume with a threshold of $40 \%$ of the SUVmax provides in some cases an aberrant volume, whereas the manual delineation actually aims to best fit the tumour's outlines. This explains the important variations in the thresholds chosen by the physicians.

Finally, our study was retrospectively conducted because of the low number of patients with oesophageal squamous cell carcinomas who are eligible for chemo radiotherapy. The study was conducted in a single centre, which may be considered to be a limitation, but it enabled us to constitute a series of strictly consecutive patients and to reduce the acquisition and setup variability [33]. Our results should be confirmed by similar studies conducted in different centres.

In conclusion, our study showed that manually determined metabolic tumour volume is a major prognostic factor for DFS and OS in patients with oesophageal squamous cell carcinomas. High SUVmax values are paradoxically associated with a longer survival. The location also appears to affect with the prognosis. Although our study requires confirmation, this conclusion should have an important clinical impact. First, manual delineation of the metabolic tumour volume is reliable, reproducible and easily feasible and is highly correlated with the prognosis. Second, the SUVmax does not have the prognostic significance usually 
assigned to it. Volumetric measurements should be preferred to the SUVmax in routine clinical practice. 


\section{References}

1. Pennathur A, Gibson MK, Jobe BA, Luketich JD. Oesophageal carcinoma. Lancet. 2013;381:400-12.

2. Zhang Y. Epidemiology of esophageal cancer. World J. Gastroenterol. 2013;19:5598-606.

3. La situation du cancer en France en 2010. Institut National du Cancer, www.e-cancer.fr Collection Rapports \& synthèses, ouvrage collectif édité par l'INCa, Boulogne-Billancourt, novembre 2010.

4. Enzinger PC, Mayer RJ. Esophageal Cancer. New Engl. J. Med. 2003;2241-52.

5. Bedenne L, Michel P, Bouché O, Milan C, Mariette C, Conroy T, et al. Chemoradiation Followed by Surgery Compared With Chemoradiation Alone in Squamous Cancer of the Esophagus : FFCD 9102. J. Clin. Oncol. 2007;25.

6. Van Westreenen HL, Westerterp M, Bossuyt PMM, Pruim J, Sloof GW, van Lanschot JJB, et al. Systematic review of the staging performance of $18 \mathrm{~F}$-fluorodeoxyglucose positron emission tomography in esophageal cancer. J. Clin. Oncol. 2004;22:3805-12.

7. Van Vliet EPM, Heijenbrok-Kal MH, Hunink MGM, Kuipers EJ, Siersema PD. Staging investigations for oesophageal cancer: a meta-analysis. Br. J. Cancer. 2008;98:547-57.

8. Downey RJ. Whole Body 18FDG-PET and the Response of Esophageal Cancer to Induction Therapy: Results of a Prospective Trial. J. Clin. Oncol. 2003;21:428-32.

9. Omloo JMT, van Heijl M, Hoekstra OS, van Berge Henegouwen MI, van Lanschot JJB, Sloof GW. FDG-PET parameters as prognostic factor in esophageal cancer patients: a review. Ann. Surg. Oncol. 2011;18:3338-52.

10. Van de Wiele C, Kruse V, Smeets P, Sathekge M, Maes A. Predictive and prognostic value of metabolic tumour volume and total lesion glycolysis in solid tumours. Eur. J. Nucl. Med. Mol. Imaging. 2013;40:290-301.

11. Di Fiore F, Blondin V, Hitzel A, Edet-sanson A, Benyoucef A, Huet E, et al. 18Ffluorodeoxyglucose positron emission tomography after definitive chemoradiotherapy in patients with oesophageal carcinoma. Dig. Liver Dis. 2012;44:875-9.

12. Touboul E, Huguet F, Talbot J-N. Use of PET for staging, treatment evaluation, and follow-up in esophageal cancers. Cancer Radiother. 2008;12:633-9.

13. Kwee RM. Prediction of Tumor Response to Neoadjuvant Therapy in Patients with Esophageal Cancer with Methods : Results : Conclusion : Radiology. 2010;254:707-17.

14. Palie O, Michel P, Ménard J-F, Rousseau C, Rio E, Bridji B, et al. The predictive value of treatment response using FDG PET performed on day 21 of chemoradiotherapy in patients with oesophageal squamous cell carcinoma. A prospective, multicentre study (RTEP3). Eur. J. Nucl. Med. Mol. Imaging. 2013;40:1345-55. 
15. Wieder H a, Brücher BLDM, Zimmermann F, Becker K, Lordick F, Beer A, et al. Time course of tumor metabolic activity during chemoradiotherapy of esophageal squamous cell carcinoma and response to treatment. J. Clin. Oncol. 2004;22:900-8.

16. Blom RLGM, Steenbakkers IR, Lammering G, Vliegen RF a, Belgers EJ, de Jonge C, et al. PET/CT-based metabolic tumour volume for response prediction of neoadjuvant chemoradiotherapy in oesophageal carcinoma. Eur. J. Nucl. Med. Mol. Imaging. 2013;

17. Hyun SH, Choi JY, Shim YM, Kim K, Lee SJ, Cho YS, et al. Prognostic value of metabolic tumor volume measured by $18 \mathrm{~F}$-fluorodeoxyglucose positron emission tomography in patients with esophageal carcinoma. Ann. Surg. Oncol. 2010;17:115-22.

18. Mamede M, Abreu-E-Lima P, Oliva MR, Nosé V, Mamon H, Gerbaudo VH. FDG$\mathrm{PET} / \mathrm{CT}$ tumor segmentation-derived indices of metabolic activity to assess response to neoadjuvant therapy and progression-free survival in esophageal cancer: correlation with histopathology results. Am. J. Clin. Oncol. 2007;30:377-88.

19. Hatt M, Visvikis D, Pradier O, Cheze-le Rest C. Baseline ${ }^{18}$ F-FDG PET image-derived parameters for therapy response prediction in oesophageal cancer. Eur. J. Nucl. Med. Mol. Imaging. 2011;38:1595-606.

20. Zhu W-Q, Sun X, Xing L, Li M, Yue J, Qu W, et al. Oesophageal squamous cell carcinoma: relationship between fluorine-18 fludeoxyglucose positron emission tomography CT maximum standardised uptake value, metabolic tumour volume, and tumour, node and metastasis classification. Br. J. Radiol. 2012;85:e383-7.

21. Herskovic a, Russell W, Liptay M, Fidler MJ, Al-Sarraf M. Esophageal carcinoma advances in treatment results for locally advanced disease: review. Ann. Oncol. 2012;23:1095-103.

22. Vauclin S, Doyeux K, Hapdey S, Vera P. Development of a generic thresholding algorithm for the delineation of 18 FDG-PET-positive tissue : application to the comparison of three thresholding. phys. Med. Biol. 2009;54:6901-16.

23. Roedl JB, Halpern EF, Colen RR, Sahani D V, Fischman AJ, Blake M a. Metabolic tumor width parameters as determined on PET/CT predict disease-free survival and treatment response in squamous cell carcinoma of the esophagus. Mol. Imaging Biol. 2009;11:54-60.

24. Fencl P, Belohlavek O, Harustiak T, Zemanova M. The analysis of factors affecting the threshold on repeated 18F-FDG-PET/CT investigations measured by the PERCIST protocol in patients with esophageal carcinoma. Nucl. Med. Commun. 2012;33:1188-94.

25. Créhange G, Bosset M, Lorchel F, Fabrice L, Buffet-Miny J, Dumas JL, et al. Tumor volume as outcome determinant in patients treated with chemoradiation for locally advanced esophageal cancer. Am. J. Clin. Oncol. 2006;29:583-7.

26. Hatt M, Visvikis D. Prognostic value of 18 F-FDG PET image-based parameters in oesophageal cancer and impact of tumour delineation methodology. Eur. J. Nucl. Med. Mol. Imaging. 2011;38:1191-202. 
27. Levine EA, Farmer MR, Clark P, Mishra G, Ho C, Geisinger KR, et al. Predictive value of 18-fluoro-deoxy-glucose-positron emission tomography (18F-FDG-PET) in the identification of responders to chemoradiation therapy for the treatment of locally advanced esophageal cancer. Ann. Surg. 2006;243:472-8.

28. Yam P-C, Tong D, Law S. Comparisons of Sixth and Seventh Edition of the American Joint Cancer Committee Staging Systems for Esophageal Cancer. Ann. Surg. Oncol. 2013;

29. Wu N, Chen Z, Pang L, Ma Q, Chen G. Prognostic significance of lymph node characteristics on survival in esophageal squamous cell carcinomas. Wien. Klin. Wochenschr. 2013;125:26-33.

30. Situ D, Wei W, Lin P, Long H, Zhang L, Fu J, et al. Do tumor grade and location affect survival in esophageal squamous cell carcinoma? Survival analysis of 302 cases of pT3N0M0 esophageal squamous cell carcinoma. Ann. Surg. Oncol. 2013;20:580-5.

31. Bashash M, Hislop TG, Shah AM, Le N, Brooks-Wilson A, Bajdik CD. The prognostic effect of ethnicity for gastric and esophageal cancer: the population-based experience in British Columbia, Canada. BMC Cancer. 2011;11:164.

32. Eloubeidi MA, Desmond R, Arguedas MR, Reed CE WC. Prognostic Factors for the Survival of Patients with Esophageal Carcinoma in the U.S. The Importance of Tumor Length and Lymph Node Status. Cancer. 2002;95:1434-43.

33. Makris NE, Huisman MC, Boellaard R. Evaluation of strategies towards harmonization of FDG PET / CT studies in multicentre trials : comparison of scanner validation phantoms and data analysis procedures. 2013;1507-15. 


\section{Conclusion}

Nous avons montré que le volume métabolique initial est un facteur pronostique de survie. Ainsi, un volume tumoral important est prédictif d'une faible survie et un volume tumoral faible est prédictif d'une survie prolongée. Dans notre série, les patients présentant un volume tumoral supérieur à $14 \mathrm{~cm}^{3}$ avaient une médiane de survie sans maladie de 8 mois et une médiane de survie globale de 16 mois, alors que les patients présentant un volume tumoral inférieur à $14 \mathrm{~cm}^{3}$ avaient une médiane de survie sans maladie de 25 mois et une médiane de survie globale de 42 mois. Notre étude a également montré que le SUVmax était un facteur pronostique de la survie sans maladie. Mais une valeur élevée de SUVmax était prédictive d'une survie sans maladie prolongée et une valeur de SUVmax plus faible était prédictive d'une survie sans maladie plus courte. Ces résultats sont concordants avec les données des publications récentes et, bien qu'ils méritent d'être confirmés par d'autres études réalisées dans différents centres, l'impact sur la pratique courante est fort. En effet, en tant que médecin nucléaire, nous cherchons à objectiver nos descriptions en donnant dans nos comptes-rendus de TEP les valeurs de SUVmax et ce, à Rouen, depuis bientôt 10 ans. Mais ni le médecin nucléaire, ni le gastro-entérologue destinataire du compte-rendu, n'attribuaient jusqu'alors à ces valeurs de SUVmax leur véritable signification pronostique. Le volume métabolique est un paramètre plus aisé à se représenter pour les cliniciens que le SUVmax et son caractère pronostique démontré par notre étude est également plus intuitif. Compte-tenu de la reproductibilité de la détermination manuelle du volume et de la corrélation aux méthodes automatiques, Il paraît tout à fait envisageable de mentionner le volume métabolique dans les comptes-rendus de TEP pour les cancers épidermoïdes de l'œsophage au moment du bilan d'extension plutôt que la valeur de SUVmax. 


\section{Annexes 1}

Localisation tumorale

(Selon le Thésaurus National de Cancérologie Digestive, Version du $1^{\mathrm{er}}$ mars 2013)

Esophage cervical :

Du bord inférieur du cartilage cricoïde à l'entrée dans le thorax, vers $19 \mathrm{~cm}$ des arcades dentaires

Esophage thoracique :

Tiers supérieur : de l'orifice supérieur du thorax à la bifurcation trachéale, à $24 \mathrm{~cm}$ des arcades dentaires environ

Tiers moyen : sous la bifurcation trachéale, de 25 à $32 \mathrm{~cm}$ des arcades dentaires environ

Tiers inférieur, incluant l'œsophage abdominal : de 33 à $40 \mathrm{~cm}$ des arcades dentaires environ 


\section{Annexes 2}

$6^{\text {ème }}$ édition (2002) de la classification TNM de l'American Joint Committee on Cancer (AJCC) et de l'Union Internationale Contre le Cancer (UICC).

- $\mathrm{T} 0=$ pas de tumeur primitive

- $\quad$ Tis $=$ carcinome in situ

- $\quad \mathrm{T} 1=$ tumeur envahissant la lamina propria ou la sous-muqueuse

- $\quad \mathrm{T} 2$ = tumeur envahissant la musculeuse propre

- $\quad$ T3 = tumeur envahissant l'adventice

- $\mathrm{Nx}=$ ganglions non évaluables

- $\quad \mathrm{N} 0=$ pas de signes d'atteinte des ganglions régionaux

- $\quad \mathrm{N} 1=$ métastases ganglionnaires lymphatiques régionales

- cervicaux pour les cancers cervicaux

- péri-œsophagiens médiastinaux et péri-gastriques (sauf cœliaques)

pour les cancers thoraciques

- $\mathrm{M} 0=$ pas de métastase à distance

- $\mathrm{M} 1=$ présence de métastase à distance

- M1a = ganglions cœliaques ou cervicaux (œsophage thoracique)

- M1b = autres sites

La classification utilisée pour ce travail est la classification de 2002 ( $6{ }^{\text {ème }}$ édition UICC)

Cette classification TNM a fait l'objet d'une actualisation en 2009 conduisant à la rédaction de la $7^{\text {ème }}$ édition qui doit dorénavant être utilisée pour la pratique. 


\section{RÉSUMÉ}

Objectifs : La TEP FDG semble avoir un intérêt pronostic dans le cancer de l'œsophage traité. Toutefois les résultats de la littérature sont discordants. Le but de cette étude était de déterminer si les paramètres métaboliques quantitatifs pré-thérapeutiques peuvent être prédictifs de la survie des patients.

Matériels et méthodes : soixante-sept patients avec un diagnostic histologique de cancer épidermoïde de l'œsophage ont été inclus dans l'étude. Chaque patient a bénéficié d'une TEP FDG avant une radio-chimiothérapie selon le protocole d'Herskovic. L'analyse quantitative a porté sur les paramètres suivants : âge, perte de poids, localisation, stade N, stade OMS, $\mathrm{MTV}_{\mathrm{p}}$ et $\mathrm{MTV}_{\mathrm{p}}$, (volume métabolique tumoral défini manuellement par deux médecins différents), $\mathrm{MTV}_{40 \%}$ (volume pour un seuil à 40\% du SUVmax), MTV automatiquement par méthode de seuillage adaptatif), SUVmax, SUVmean et TLG.

Résultats : Le $\mathrm{MTV}_{\mathrm{p}}$ et le $\mathrm{MTV}_{40 \%}$ étaient fortement corrélés (index de Pearson 0,92). Le $\mathrm{SUVmean}_{\mathrm{p}}$ et $\mathrm{SUVmean}_{40}$ étaient également corrélés (index de Pearson 0,86). Le TLG $\mathrm{T}_{\mathrm{p}}$ et le $\mathrm{TLG}_{40 \%}$ étaient corrélés (index de Pearson 0,98). De la même façon les paramètres issus de la méthode seuillage adaptatif $\left(\mathrm{MTV}_{\mathrm{a}}, \mathrm{SUVmean}_{\mathrm{a}}\right.$ et $\left.\mathrm{TLG}_{\mathrm{a}}\right)$ étaient corrélés aux paramètres issus de la délinéation manuelle $\left(\mathrm{MTV}_{\mathrm{p}}, \mathrm{SUVmean}_{\mathrm{p}}\right.$ et $\left.\mathrm{TLG}_{\mathrm{p}}\right)$. La détermination manuelle du volume tumoral $\left(\mathrm{MTV}_{\mathrm{p}}\right.$ et $\mathrm{MTV}_{\mathrm{p}}$ ) était reproductible (index de Pearson 0,95). L'analyse multi-variée portant sur la survie sans maladie (DFS) retrouvait qu'un $M_{T} V_{p}$ élevé était associée à une courte DFS $(\mathrm{p}=0,004)$ et qu'un SUVmax élevé était associée à une longue DFS $(\mathrm{p}=0,02)$. Les autres paramètres n'étaient pas statistiquement significatifs. L'analyse multivariée portant sur la survie globale (OS) retrouvait qu'un $M_{T} V_{p}$ élevé était associée à une courte $\mathrm{OS}(\mathrm{p}=0,01)$, et qu'une localisation au tiers inférieur était associée à une longue OS $(\mathrm{p}=0,005)$. Les autres paramètres n'étaient pas statistiquement significatifs.

Conclusion : Le volume métabolique tumoral initial est prédictif de la survie sans maladie et de la survie globale chez les patients présentant un carcinome épidermoïde œsophagien. Un SUVmax élevé est paradoxalement associé à une survie prolongée. La localisation semble également intervenir dans le pronostic.

\section{MOTS CLEFS}

TEP FDG - paramètres métaboliques quantitatifs - volume métabolique tumoral - survie Cancer épidermoïde de l'œsophage - radio-chimiothérapie 